\title{
EL PROBLEMA DE LA ESTABILIDAD
}

Hugo Seleme
Universidad Nacional de Córdoba, Argentina

\section{Introducción}

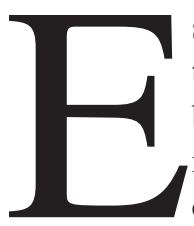

s sobradamente conocido que los cambios más importantes introducidos por Rawls a su «Teoría de la Justicia» ${ }^{1}$, con la publicación de «Liberalismo Político»², tienen su origen en la manera novedosa en que explica allí la estabilidad de una concepción política de la justicia 3 .

Rawls mismo ha dejado en claro desde las primeras páginas de esta obra que la mayoría de las alteraciones realizadas a la versión original de su teoría se deben a que la explicación de la estabilidad que se brindaba en «Teoría de la Justicia» era inadecuada. Así señala que las diferencias entre una obra y otra surgen:

«...del intento de resolver un serio problema inherente a la justicia como imparcialidad; a saber, el que surge del hecho de que la explicación de la estabilidad en la Tercera Parte de la Teoría de la Justicia no es congruente con el panorama global. Pienso que todas las diferencias en este aspecto son consecuencia del intento de disipar tal incongruencia...» ${ }^{4}$

En lo que sigue me propongo analizar algunas de las objeciones que han sido formuladas en contra de las ideas expresadas por Rawls en su nueva ex-

\footnotetext{
${ }^{1}$ Rawls, John; Teoría de la Justicia, Fondo de Cultura Económica, $1^{\text {a }}$ reimpresión, 1997.

${ }^{2}$ Rawls, John; Liberalismo Político, Fondo de Cultura Económica, 2a reimpresión, 1996.

${ }^{3}$ La estabilidad de una concepción política es distinta a la estabilidad de un régimen político. Un régimen democrático es estable si es apoyado voluntariamente por la mayoría de sus ciudadanos políticamente activos. Una concepción política de justicia es estable si aquellos que crecen en una sociedad bien ordenada de acuerdo a sus principios desarrollan un suficientemente fuerte sentido de justicia, de manera que actúen normalmente según sus requerimientos, siempre y cuando sus conciudadanos hagan lo mismo. Cfr. Rawls, John; «The Domain of the Political and Overlapping Consensus», en «Collected Papers» ed. Samuel Freeman, Harvard University Press: (473-496) pág. 479.

4 «Liberalismo Político» Op. cit.pág. 11.
} 
posición de la justicia como imparcialidad. Estas objeciones han sido sostenidas por Brian Barry en su artículo «John Rawls and the Search for Stability» ${ }^{5}$ publicado en Ethics dos años después de la aparición de «Liberalismo Político».

En él, Barry dirige dos tipos de argumentos en contra de Rawls. Unos apuntan a mostrar que «Liberalismo Político» le atribuye a «Teoría de la Justicia» errores que esta no contiene. Otros tienen por objeto establecer que «Liberalismo Político» en sí mismo contiene errores.

El objetivo de este trabajo es discutir algunos de ambos tipos de argumento.

Así en la primera parte del trabajo expondré dos de los argumentos que Barry dirige en contra de Rawls para fundar su afirmación de que «Liberalismo Político» le atribuye a «Teoría de la Justicia» errores que esta no contiene. Intentaré mostrar que los errores que Rawls señala a la versión original de su teoría son reales y que los argumentos utilizados para mostrar lo contrario son inválidos.

En la segunda parte del trabajo me ocuparé de las críticas que Barry dirige en contra de «Liberalismo Político» en sí mismo, especialmente la que sostiene que, a fin de explicar la estabilidad, la apelación al recurso del «consenso superpuesto» de doctrinas razonables es innecesaria.

Reconstruiré de manera abreviada la principal línea argumental que lo lleva a Barry a sostener esta conclusión para luego ocuparme de analizarla. En especial intentaré mostrar por qué, en función de los supuestos básicos de la teoría de Rawls, el argumento no es correcto.

\section{- 1 -}

1.1- Barry acuerda con Rawls que los cambios introducidos en «Liberalismo Político» a la concepción de la justicia como imparcialidad están relacionados con el problema de la estabilidad. Sin embargo piensa que los problemas que Rawls ve en «Teoría de la Justicia» no son tales.

Así, no está de acuerdo con Rawls en relación a que:

a) La concepción expuesta en «Teoría de la Justicia» constituya una «doctrina filosófica comprehensiva» o forme parte de una doctrina de este tipo.

b) La concepción de una «sociedad bien ordenada», tal como se la expone allí, implique la aceptación por parte de todos los ciudadanos de una «doctrina filosófica comprehensiva». ${ }^{6}$

\footnotetext{
${ }^{5}$ Barry, Brian; «John Rawls and the Search for Stability», Ethics 105 (Julio 1995): 874-915.

${ }^{6}$ Señala Rawls haciendo referencia a los dos problemas señalados «...el serio problema al que me he referido es el de la idea poco realista de una sociedad bien ordenada, tal como aparece en mi Teoría de la Justicia. Una característica esencial de una sociedad bien ordenada, aquella que podamos relacionar con la justicia como imparcialidad, es que todos sus ciudada-
} 
1.2- Antes de exponer el argumento de Barry para sustentar su pretensión de que «Teoría de la Justicia» no constituye una «doctrina filosófica comprehensiva», es necesario aclarar qué es lo que Rawls critica a la exposición de su doctrina efectuada en aquella obra.

Pienso que uno de los elementos que Rawls encuentra objetables en «Teoría de la Justicia» es que la exposición de la justicia como imparcialidad es ambigua ${ }^{7}$. Estando ausente la distinción entre una «doctrina comprehensiva» y una «concepción política», la justicia como imparcialidad puede ser interpretada de las dos maneras, esto es, como parte de una «doctrina comprehensiva» o como una «concepción política».

El problema, sin embargo, no es sólo de ambigüedad. Así señala Rawls en «The Domain of The Political and Overlapping Consensus» ${ }^{8}$ que existen pasajes de «Teoría de la Justicia» que pueden hacer que un lector razonable concluya que la justicia como imparcialidad es parte de una visión comprehensiva.

Estos pasajes son:

a) La afirmación de Rawls en el sentido de que si la justicia como imparcialidad tenía éxito el paso siguiente sería estudiar la concepción más general sugerida por el nombre «la rectitud como imparcialidad». ${ }^{9}$

b) La discusión de la sociedad bien ordenada según la justicia como imparcialidad, en la cual se suponía que sus miembros aceptaban la misma concepción de justicia en tanto aceptaban la misma doctrina comprehensiva de la cual aquella concepción era parte, o a partir de la cual podía ser derivada $^{10}$.

nos respaldan este concepto basándose en lo que ahora llamamos una doctrina comprehensiva filosófica. Todos los ciudadanos aceptan, como enraizados en esta doctrina, sus dos principios de justicia...Aunque no se analiza en mi Teoría de la Justicia la distinción entre una concepción política de la justicia y una doctrina comprehensiva filosófica, una vez que se ha planteado esta cuestión queda claro, a mi parecer, que el texto considera tanto a la justicia como imparcialidad y al utilitarismo como doctrinas comprehensivas, o parcialmente comprehensivas». Liberalismo Político Op. cit., pág. 11.

7 Uno de los objetivos de Liberalismo Político es subsanar esta ambigüedad. «...Desaparece ahora la ambigüedad propia de mi Teoría de la justicia, y desde el comienzo la justicia como imparcialidad se presenta como una concepción política de la justicia» (el subrayado me pertenece), Ibid. pág. 12 .

8 «The Domain of the Political and Overlapping Consensus», Op. cit. Los mismos errores atribuibles a Teoría de la Justicia han sido señalados por Rawls en la última exposición que ha hecho de su concepción política de la justicia. Rawls, John; «Justice as Fairness: A Restatement», ed.Erin Kelly, Harvard University Press, 2001, pág. 186-187.

${ }^{9}$ Cfr. Ibid. pág. 488-489, y Teoría de la Justicia, Op.cit, pág. 29.

10 Cfr. Ibid pág. 489, y Teoría de la Justicia, Op. cit., Parte III pág. 359 y ss. 
El primer problema que Rawls ve en su «Teoría de la Justicia» no es, entonces, que la justicia como imparcialidad, dejando de lado su explicación de la estabilidad, sea una doctrina comprehensiva, sino que es presentada allí como parte de una doctrina que sí lo es, a saber, «la rectitud como imparcialidad».

Rawls piensa que es el carácter general de la «rectitud como imparcialidad» lo que hace de ella una doctrina comprehensiva. Así, señala en «Teoría de la justicia»:

«...si la justicia como imparcialidad tiene un éxito razonable, el siguiente paso sería estudiar la concepción más general sugerida por el nombre "la rectitud como imparcialidad". Pero incluso esta teoría más amplia no abarcaría todas las relaciones morales, ya que parecerá incluir sólo nuestras relaciones con otras personas, dejando sin explicar cómo habremos de conducirnos respecto a los animales y al resto de la naturaleza» (el subrayado me pertenece) $)^{11}$.

y refiriéndose a este pasaje agrega en «The Domain of the Political and Overlapping Consensus»:

«...El lector puede razonablemente concluir, entonces, que la justicia como imparcialidad es presentada como parte de una visión comprehensiva que puede ser desarrollada más tarde...» (el subrayado me pertenece) $)^{12}$.

Es necesario entonces analizar qué relación existe entre el carácter general y la comprehensividad de una concepción, de manera que si una concepción tiene el primero entonces también tiene el segundo.

Una doctrina es general:

«...cuando se aplica a un amplio espectro de materias (en el límite extremo, a todos los temas)...» ${ }^{13}$.

Una doctrina no es general, por el contrario, cuando ha sido elaborada, y se aplica sólo a una materia específica.

Una doctrina es comprehensiva:

«..cuando incluye concepciones de lo que se considera valioso para la vida humana, así como ideas de virtudes y carácter personales, que conformarán gran parte de nuestra conducta no política (en el límite extremo, toda nuestra vida) $»^{14}$.

A su vez una doctrina comprehensiva puede ser plenamente comprehensiva:

«...cuando abarca todos los valores y virtudes reconocidas dentro de un esquema de pensamiento más o menos bien articulado...» ${ }^{15}$.

${ }^{11}$ Rawls, John; Teoría de la Justicia Op. cit., pág. 29.

${ }^{12}$ Rawls, John; «The Domain of the Political and Overlapping Consensus», Op. cit., pág. 489.

${ }^{13}$ Rawls, John; Liberalismo Político, Op. cit., pág. 172.

${ }^{14}$ Ibid.

${ }^{15}$ Ibid. 
o parcialmente comprehensiva:

«...cuando abarca ciertos valores no políticos (mas no todos) y sólo algunas virtudes, y está articulada de manera laxa... $\gg^{16}$.

Por otro lado, una doctrina no es comprehensiva cuando es política, esto es, cuando se aplica sólo a una materia específica: las instituciones sociales, económicas y políticas básicas, y abarca ciertos valores, e ideas de virtud y carácter personal, que sólo son aplicables a las conductas políticas ${ }^{17}$.

Una concepción política, a su vez, puede ser también completa o parcial según que se aplique a todas las instituciones sociales, económicas y políticas básicas, o sólo a alguna de ellas. ${ }^{18}$

La «justicia como imparcialidad», tal como se la presenta en «Liberalismo Político», en tanto concepción aplicable sólo a la estructura básica de una sociedad doméstica, sería una parcial concepción política de justicia.

Esta concepción, junto con la concepción de justicia presente en «The Law of Peoples» ${ }^{19}$, la cual tiene por objeto el derecho y las prácticas de la sociedad de pueblos organizados políticamente, forman una más completa concepción política de la justicia ${ }^{20}$.

\section{Ibid.}

17 Una dificultad con la clasificación que propongo es que Rawls no diferencia las doctrinas comprehensivas de las concepciones políticas en general, sino de una concepción política de la justicia. El problema sería el siguiente, en primer lugar: ¿existen otras concepciones políticas que no se refieran a la justicia? y, en segundo lugar, si existen, ¿las características que diferencian según Rawls a una concepción política de la justicia de una visión comprehensiva son compartidas por todas las concepciones políticas estén o no referidas a la justicia? Dejando de lado el primer problema en el texto he supuesto que aun si existieran otras concepciones políticas aquello que las diferenciaría de una doctrina comprehensiva sería lo mismo que diferencia a estas de una concepción política de la justicia. Apoyando esta interpretación puede utilizarse el hecho de que Rawls en «The Domain of the Political and Overlapping Consensus» luego de presentar (en la sección IV) las características que diferencian a una concepción política de la justicia de una doctrina comprehensiva se refiere a estas (en la sección V) como las características de una concepción política, sin ninguna especificación. Señala en esta sección: «Las tres características de una concepción política dejan en claro que la justicia como imparcialidad no es filosofía moral aplicada...» (el subrayado me pertenece), Rawls, John; «The Domain of the Political and Overlapping Consensus», Op. cit., pág. 482. En el texto no me he pronunciado sobre la posibilidad de que existan otras concepciones políticas no referidas a la justicia, simplemente he sostenido que de existir tendrían las mismas caracterísitcas que estas.

${ }^{18}$ El término «parcial» no es utilizado por Rawls, sino que simplemente habla de concepciones políticas «más completas» por lo que el rótulo más adecuado parecería ser el de «menos completas». Sin embargo utilizar la palabra «parcial» me ha parecido útil para mantener la analogía con la clasificación de las doctrinas comprehensivas.

19 Rawls, John; «The Law of Peoples», en Freeman, Samuel (ed); Collected Papers, pág. 529-564.

${ }^{20} \mathrm{Al}$ respecto señala Rawls en la última presentación de su concepción: «...Las dos partes de una concepción política más completa -la justicia de la sociedad doméstica así como (la justicia) de las relaciones entre sociedades- pueden ser ajustadas una a la otra en el proceso de 
Ahora bien ¿de qué manera están interrelacionadas estas clasificaciones?

En primer lugar, no toda concepción específica, es decir no general, es política. Esto en tanto que puede haber sido elaborada para una materia específica distinta a las instituciones sociales, económicas y políticas básicas. Tal sería el caso de una doctrina que hubiera sido elaborada, y sólo fuera aplicable, a la conducta sexual. En este caso estaríamos frente a una doctrina no general y comprehensiva.

En segundo lugar, toda concepción política, en tanto elaborada para una materia específica, no puede ser general.

En último término, y lo que interesa a nuestros fines, toda concepción general no es política. Esto debido a que si se aplica a un amplio espectro de temas entonces excede el ámbito de lo político, entendido como las instituciones sociales, económicas y políticas básicas. Por otro lado, que su ámbito de aplicación exceda lo político exige que su contenido sea más amplio $^{21}$, esto es, que contenga valores, e ideas de virtud y carácter personal, no sólo referidos ya al ámbito de las instituciones básicas, es decir, a las conductas políticas, sino referidos a las conductas que exceden ese marco. De esto se sigue, entonces, que toda concepción general es comprehensiva.

Puesto que la «justicia como imparcialidad» era presentada en «Teoría de la Justicia» como parte de una visión más general, entonces, un lector razonable podía concluir que era parte de una visión comprehensiva tal como la «corrección como imparcialidad».

1.3- Pienso que Barry no comparte esta conclusión porque está en desacuerdo con la idea de Rawls de que toda doctrina general, esto es que no tenga por objeto las principales instituciones políticas, sociales y económicas, sea comprehensiva.

Una doctrina tal como la «corrección como imparcialidad» no sería política, sostiene Barry, debido a que su contenido no sería un diseño de las instituciones sociales básicas, sino reglas morales. Pero agrega, siendo esta doctrina una extensión de la «justicia como imparcialidad» sólo poseería reglas diseñadas para permitir a las personas, con diferentes concepciones del

\footnotetext{
elaborarlas» (el subrayado me pertenece). Rawls, John; «Justice as Fairness: A Restatement», Op. cit., pág. 14.

${ }^{21}$ Respecto a la interrelación entre generalidad, la más amplia gama de temas a los que se aplica una concepción, y comprehensividad señala Rawls: «...la distinción entre las concepciones políticas de la justicia y otras concepciones morales es un asunto de perspectiva: esto es, que se refiera a la gama de temas a los que se aplica una concepción, y al más amplio contenido que exige una gama de temas más amplia...» (el subrayado me pertenece). Rawls, John; Liberalismo Político,Op. cit. , pág. 172.
} 
bien, vivir juntas, es decir poseería reglas que prescribirían límites a las acciones permisibles antes que reglas que establecieran fines sustantivos ${ }^{22}$.

La idea de Barry es que la única diferencia que existiría entre la «justicia como imparcialidad» y una concepción más general tal como «la corrección como imparcialidad», sería que su contenido no serían las instituciones básicas, sino reglas morales. Estas reglas, al igual que aquellas instituciones, estarían diseñadas para permitir diferentes concepciones del bien en los ciudadanos. En consecuencia, si la «justicia como imparcialidad» no es una doctrina comprehensiva, tampoco lo es la «corrección como imparcialidad» ${ }^{23}$.

Barry sostiene, entonces, que la «corrección como imparcialidad», por un lado, tendría el mismo objeto que la «justicia como imparcialidad»: organizar la convivencia política entre personas con distintas concepciones del bien; y por otro, tendría su mismo carácter deontológico. Su única diferencia es que contendría en lugar de instituciones, reglas morales.

Ahora bien, como estas reglas formarían parte de una concepción deontológica, antes que establecer fines sustantivos a los que considerar valiosos, tal como según Rawls haría una doctrina comprehensiva, prescribirían límites a las acciones permisibles.

1.4- Lo primero que me parece errado en la idea de Barry es su forma de presentar la «corrección como imparcialidad». El objeto de una doctrina moral que abarque todas nuestras relaciones con otras personas ${ }^{24}$ no puede ser simplemente, como este sostiene, la convivencia política.

Una concepción como la «corrección como imparcialidad» consistiría, o por lo menos incluiría, una concepción general de justicia. Contendría por un lado una concepción de justicia doméstica tal como la «justicia como imparcialidad», por otro, una concepción de justicia internacional tal como la que Rawls presenta en «The Law of Peoples», y finalmente, contendría una concepción de justicia local que se aplique a la vida interna de las asociaciones e instituciones de la sociedad civil.

Pienso que es este último componente de una concepción general de justicia el que la hace una doctrina comprehensiva. Señala Rawls:

«...debemos concentrarnos en la estructura básica como el tema primario de la justicia política y dejar de lado cuestiones de justicia local. No vemos a la justicia como imparcialidad como una doctrina moral comprehensiva sino como

22 Cfr. «Rawls and the Search for Stability», supra nota 5, pág. 878.

23 Señala Barry «...Una teoría rawlsiana de «corrección como imparcialidad» desarrollada como una extensión de la «justicia como imparcialidad» estaría interesada entonces con lo correcto más que con lo bueno. En término de Rawls, sería una concepción política...». Ibid. pág. 879.

${ }^{24}$ Cfr. Rawls, John; Teoría de la Justicia, Op. cit., pág. 29 
una concepción política a aplicarse a aquella estructura de instituciones políticas y sociales» (el subrayado me pertenece ${ }^{25}$.

Contrario sensu, una concepción general de justicia que incluyera cuestiones de justicia local sería, según Rawls, una doctrina comprehensiva.

La razón de esto es clara. Toda concepción moral, incluida la «justicia como imparcialidad», contiene valores e ideales de virtud y carácter personal. Sin embargo esto no la vuelve una doctrina comprehensiva, porque el ámbito de aplicación de estos es únicamente la conducta política.

El incluir valores, ideales y virtudes no es lo que distingue a una doctrina deontológica de una teleológica, sino sólo, la manera de derivarlos y justificarlos, otorgando o no prioridad a lo correcto sobre lo bueno.

Así, la «justicia como imparcialidad», aun siendo una doctrina deontológica, contiene valores tales como, el de la igual libertad y los bienes primarios, ideales, como la concepción de persona y de sociedad bien ordenada, y virtudes, como el poseer un sentido de la justicia ${ }^{26}$.

Ahora bien, si la concepción es vista como parte de una visión más general estos valores, ideales y virtudes dejan de aplicarse simplemente a las conductas políticas y pasan a aplicarse a las relaciones humanas en general, con lo cual la concepción más general se transforma en una visión comprehensiva.

Algunas de las objeciones que ha recibido la concepción rawlsiana de justicia han estado fundadas en esta interpretación general de su ámbito de aplicación. Así Sandel, por ejemplo, plantea una situación familiar donde los vínculos afectivos son reemplazados por demandas de imparcialidad y observancia de los derechos. Este es el único ambiente familiar, sostiene Sandel, en donde los principios de justicia podrían ser realizados. Más aún, continúa el argumento, bastaría que los principios de justicia se aplicaran a las relaciones familiares para que la situación se transformara en la antes descripta. Esto porque cuando alguien actúa guiado por un sentido de la justicia en circunstancias donde prevalecen los lazos benevolentes y afectivos, su actuar de esta manera contribuye a quebrantar estos vínculos, de la misma manera que demostraciones físicas de valentía en medio de un ámbito de tranquilidad pueden contribuir a viciarlo ${ }^{27}$.

La «justicia como imparcialidad» implicaría un ideal de vida familiar y, en consecuencia, sería una visión comprehensiva. Esto en tanto este ideal o modelo se aplica a conductas que no son políticas ${ }^{28}$.

\footnotetext{
25 Rawls, John; «Justice as Fairness: A Restatement», Op. cit., pág. 12.

${ }^{26}$ Cfr. Rawls, John; Teoría de la Justicia, Op. cit., pág. 184, 395-396.

27 Sandel, Michael, Liberalism and the Limits of Justice, Cambridge University Press, (1982), sec. ed. (1998), pág. 33-35.

28 En igual sentido se pronuncia Sandel en Democracy's Discontent: America in Search of a Public Philosophy, Harvard University Press, (1998), Cfr. pags. 108-119.
} 
La réplica de Rawls a esta objeción es iluminadora, porque muestra la vinculación que existe entre el carácter general de una concepción de la justicia y su carácter comprehensivo. Señala, en relación a Sandel:

«..Un error aquí es que él supone que los dos principios se aplican en general a todas las asociaciones, mientras que ellos se aplican sólo a la estructura básica...Alguna concepción de justicia es en verdad vista como apropiada para la familia, así como para otras asociaciones y casos de justicia local...» (el subrayado me pertenece $)^{29}$.

Es útil notar que Rawls no cuestiona el argumento de Sandel señalando que si los principios de justicia se aplican a la vida familiar esto implica adoptar una visión comprehensiva, sino que, por el contrario, su réplica es que los principios no se aplican a este ámbito, así como a ninguno que involucre la vida interna de las asociaciones. Esto es objeto de las concepciones de justicia local, no de las concepciones políticas de la justicia.

Aunque la réplica de Rawls muestra que la «justicia como imparcialidad» no es, en sí misma, una visión comprehensiva, deja claro que la manera en que se la presentaba en «Teoría de la Justicia» como parte de una concepción más general era defectuosa. Esto porque esta concepción más general, cuyo objeto eran todas las relaciones humanas, tendría que incluir una concepción general de justicia, la cual abarcaría concepciones de justicia local, las que, a su vez, contendrían valores e ideales de virtud y carácter personal que se aplicarían a la vida interna de las asociaciones y, por tanto, regularían las conductas no políticas de sus miembros. Es decir, esta concepción general, de la cual la «justicia como imparcialidad» se presentaba como una parte, sería una visión comprehensiva.

1.5- La segunda tesis de Barry tiende a controvertir la afirmación de Rawls con relación a que la idea de una sociedad bien ordenada, tal como se la expone en «Teoría de la Justicia», implica la aceptación por parte de todos los ciudadanos de una «doctrina filosófica comprehensiva».

Para defender la tesis que sostiene que Rawls está equivocado al hacer esta afirmación su estrategia es la siguiente: mostrar la idea de «sociedad bien ordenada» que está presente en «Liberalismo Político» y compararla con la que se encuentra en «Teoría de la Justicia» a fin de mostrar que son idénticas. La conclusión es que dado que la manera de concebir una «sociedad bien ordenada» tal y como se la presenta en «Liberalismo Político» no implica, según Rawls, la aceptación de ninguna «doctrina comprehensiva» por parte de los ciudadanos, entonces la concepción presentada en «Teoría de la Justicia» tampoco lo hace.

${ }^{29}$ Rawls, John; «Justice as Fairness: A Restatement», Op. cit., pág. 165, nota 48. 
Lo único que debe ser probado para que el argumento tenga éxito es la identidad de las concepciones, porque la premisa restante, esto es, que la concepción de «sociedad bien ordenada» presente en «Liberalismo Político» no requiere la aceptación por parte de los ciudadanos de ninguna doctrina comprehensiva, es algo que el mismo Rawls afirma.

La prueba que Barry ofrece se sustenta, entonces, en la comparación de ambos textos, «Teoría de la Justicia» y «Liberalismo Político». Sostiene que si los criterios utilizados para determinar cuándo una sociedad está bien ordenada son los mismos en uno y otro texto, entonces la concepción utilizada es la misma, y la conclusión se sigue.

El paso siguiente tomado por Barry es mostrar que los criterios utilizados son efectivamente los mismos, a saber: a) Todos aceptan, y saben que los demás aceptan, los mismos principios. b) Su estructura básica es públicamente conocida, o con buenas razones creída, satisfacer estos principios. c) Sus ciudadanos tienen normalmente un efectivo sentido de la justicia y obedecen en general a sus instituciones sociales básicas, a las cuales reconocen como justas. ${ }^{30}$

De la identidad de los criterios Barry concluye:

«...Dejando de lado los detalles verbales, estos son manifiestamente los mismos tres criterios. No existe sustento para el reclamo corriente de Rawls de que una sociedad bien ordenada como era concebida en Teoría de la Justicia requería que los ciudadanos apoyaran los principios de justicia sobre las bases de una doctrina filosófica comprehensiva...» (el subrayado me pertenece) ${ }^{31}$.

Pienso que el argumento de Barry es incorrecto y la razón de esto es que se asienta en una confusión entre concepto y concepción ${ }^{32}$.

En efecto, de que el concepto de «sociedad bien ordenada» sea el mismo tanto en «Teoría de la Justicia» como en «Liberalismo Político», Barry extrae la conclusión de que las concepciones presentes en ambas obras son idénticas, y de esta manera obtiene su conclusión ${ }^{33}$.

${ }^{30}$ Para ver la presentación de estos tres requisitos de una sociedad bien ordenada Cfr. Rawls, John; Teoría de la justicia, Op. cit., pág. 410-418 y Liberalismo Político, Op. cit., pág. 56-60.

31 Barry, Brian; «John Rawls and the Search for Stability», Op. cit., pág. 879.

32 Rawls utiliza la distinción entre concepto y concepción en «Teoría de la Justicia» para distinguir entre el concepto de justicia y las diferentes concepciones en relación al mismo. Cfr supra. nota 1 pág. 19. Cfr. Dworkin, Ronald; El Imperio de la Justicia, Edit. Gedisa, $2^{\mathrm{a}}$ Ed. 1992, pág. 74-83.

${ }^{33}$ En apoyo de la confusión en que sostengo incurre Barry puede señalarse su afirmación al comenzar el argumento sosteniendo: «Hemos visto que en "Teoría de la Justicia" Rawls hace uso de la idea de una "sociedad bien ordenada" para realizar su contraste entre lo correcto (sobre lo cual es requerido acuerdo) y lo bueno (sobre lo que no es requerido). Curiosamente, sin embargo, él invoca precisamente este concepto en "Liberalismo Político" en el curso de ex- 
Ahora bien, por supuesto que Rawls no está disconforme con el «concepto» de «sociedad bien ordenada» utilizado en «Teoría de la Justicia», sino con la particular manera en que se lo entendía o interpretaba en esa obra.

Que los criterios para establecer cuándo una sociedad está «bien ordenada» sean idénticos en las dos obras lo único que muestra es que en ambos casos se está hablando de lo que es una «sociedad bien ordenada», y consiguientemente el concepto utilizado es el mismo. Pero de aquí no se sigue, como Barry pretende, que la manera de entender lo que la existencia de una «sociedad bien ordenada» requiere, esto es, su concepción, sea igual.

Para ejemplificar que el acuerdo en el «concepto» no implica acuerdo en la «concepción» basta con señalar el caso de las diferentes concepciones de justicia tratado por el mismo Rawls. Así, tanto el utilitarismo como la justicia como imparcialidad estarían de acuerdo en que las instituciones justas son aquellas que no realizan distinciones arbitrarias para la asignación de derechos y deberes. Esto, señala Rawls, sería parte del acuerdo sobre el concepto de justicia. Sin embargo, parecería extraño extraer de este acuerdo en relación al concepto, la conclusión de que tanto el utilitarismo como la justicia como imparcialidad entienden la arbitrariedad de la misma manera, y consecuentemente, tienen a este respecto la misma concepción de justicia.

Este paso indebido, desde el acuerdo respecto al concepto a la identidad de la concepción, es el realizado por Barry en relación a la idea de «sociedad bien ordenada».

Para explorar si las concepciones de «sociedad bien ordenada» presentes en «Teoría de la Justicia» y «Liberalismo Político» son las mismas no es suficiente constatar que los requisitos son literalmente los mismos, resta además constatar que son interpretados en ambas obras de la misma manera.

Lo dicho basta para mostrar que el argumento de Barry es inválido, ya que, del hecho de que el concepto de «sociedad bien ordenada» usado en «Teoría de la Justicia» y «Liberalismo Político» sea el mismo, no se sigue que sus concepciones sean idénticas, y esto es lo que hay que mostrar si el argumento ha de tener éxito.

Sin embargo, como es obvio, que el argumento para sostener la premisa que afirma la identidad de las concepciones sea inválido no basta para sostener que esta es falsa. Mostrar esto es el próximo paso.

1.6- Al presentar el problema en «Liberalismo Político» Rawls señala que la raíz de este se halla en la explicación defectuosa de la estabilidad que

plicar qué es lo que ahora piensa que está equivocado en "Teoría de la Justicia"...»; para concluir luego: «....No existe sustento para la pretensión corriente de Rawls de que una sociedad bien ordenada tal como era concebida en "Teoría de la Justicia" requería de los ciudadanos aceptar los principios de justicia en base a una doctrina filosófica comprehensiva». Barry, Brian; «John Rawls and the Search for Stability», Op. cit., pág. 879. 
se encuentra en «Teoría de la Justicia». Señala Rawls refiriéndose a la causa de las modificaciones introducidas en «Liberalismo Político»:

«...surgen del intento de resolver un serio problema inherente a la justicia como imparcialidad; a saber, el que surge del hecho de que la explicación de la estabilidad en la Tercera Parte de la Teoría de la justicia no es congruente con el

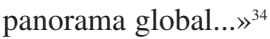

y luego agrega con relación al párrafo precedente:

«...el serio problema al que me he referido es el de la idea poco realista de una sociedad bien ordenada, tal como aparece en mi Teoría de la Justicia...» ${ }^{35}$

Pero ¿de qué manera están relacionadas la explicación de la estabilidad y la de una «sociedad bien ordenada»? ¿Qué clase de relación es esta que produce que si la explicación de la primera es defectuosa la explicación de la segunda también lo sea?

Si entiendo bien el pensamiento de Rawls, la explicación de la estabilidad es referida por el concepto de «sociedad bien ordenada» de manera que aunque sus requisitos permanezcan idénticos, si la concepción de la estabilidad cambia, lo mismo sucede con la concepción, esto es con la manera de interpretar el concepto, de «sociedad bien ordenada». Para decirlo de otra manera, la interpretación que se dé a los requisitos para que exista una «sociedad bien ordenada» depende de la concepción de la estabilidad que se tenga.

La idea sería la siguiente. Uno de los requisitos para que exista una «sociedad bien ordenada» es que los ciudadanos tengan un suficientemente fuerte sentido de la justicia, de tal manera que ellos normalmente actúen como la justicia requiere, siempre y cuando estén seguros de que otros actuarán de igual manera ${ }^{36}$ Este requisito remite a la motivación que deben tener los ciudadanos razonables para obedecer dentro de una «sociedad bien ordenada».

A su vez el que los ciudadanos que han crecido bajo instituciones regidas por una concepción política de la justicia tengan un fuerte y normalmente efectivo deseo de obedecer es condición suficiente, según Rawls, para que aquella concepción política sea estable. Es decir, entonces, que una «sociedad bien ordenada» no puede ser tal, a menos que la concepción política de la justicia que la rija sea estable. En consecuencia las condiciones suficientes para la estabilidad de una concepción política, y la manera de entenderlas y alcanzarlas, son condiciones necesarias de la «sociedad bien ordenada».

\footnotetext{
34 Rawls, John; Liberalismo Político, Op. cit., pág. 11.

35 Ibid.

${ }^{36}$ Cfr. Ibid. pag 56 y ss, y Rawls, John, Teoría de la Justicia, Op. cit., pág. 410-418.
} 
Esto da cuenta de la afirmación de Rawls en el sentido de que si la manera en que se explica la estabilidad de la concepción política de la justicia, esto es la forma en que los ciudadanos que crecen en una sociedad ordenada por aquella concepción pueden adquirir un fuerte y normalmente efectivo deseo de actuar conforme a sus exigencias, es poco realista, entonces la idea de «sociedad bien ordenada» también lo es.

De igual modo, si la forma en que se explica la adquisición de esta motivación por parte de los ciudadanos varía, esto es si varía la concepción de estabilidad, entonces la concepción de «sociedad bien ordenada» también lo hace. Aunque el requisito permanece idéntico, la manera de interpretarlo y, por tanto, de satisfacerlo cambia.

Barry reconoce que lo que está mal en «Teoría de la Justicia» es la explicación que allí se da de la estabilidad al señalar:

«...podríamos decir que, aunque la teoría de justicia en sí misma no depende de una «visión comprehensiva», la explicación de su estabilidad si lo hace.»»

Ahora bien, si lo sostenido antes es acertado, y si una condición necesaria para que la concepción política de la justicia como imparcialidad sea estable es que aquellos ciudadanos de una sociedad ordenada por ella acepten una visión comprehensiva, entonces esto último es también una condición necesaria para que exista una «sociedad bien ordenada» por la justicia como imparcialidad.

Dicho sintéticamente, si la aceptación de una doctrina comprehensiva por parte de los ciudadanos es una condición necesaria de la estabilidad de una concepción política, tal y como se la entendía en «Teoría de la Justicia», y si la estabilidad de la concepción política es una condición necesaria para que exista una «sociedad bien ordenada», entonces lo primero es una condición necesaria de lo último.

Como la manera de entender la estabilidad, según el mismo Barry reconoce, ha variado de «Teoría de la Justicia» a «Liberalismo Político», la concepción de «sociedad bien ordenada», debe reconocer, también lo ha hecho.

Si las concepciones no son idénticas el argumento de Barry sosteniendo que, dado que la idea de «sociedad bien ordenada» en «Liberalismo Político» no implica la aceptación de ninguna doctrina comprehensiva, la idea de «sociedad bien ordenada» presente en «Teoría de la Justicia» tampoco lo hace, es inválido.

Más aun, Barry no sólo debería reconocer ahora que el pasar de la identidad de concepto a la identidad de concepción es inválido, sino que ade-

${ }^{37}$ Barry, Brian; «John Rawls and the Search for Stability», Op. cit., pág. 887. 
más, y debido al segundo argumento presentado, debería reconocer la falsedad de aquella premisa.

Es decir entonces que el argumento es incorrecto por dos motivos, en primer lugar porque realiza un paso indebido de la identidad de conceptos a la identidad de concepciones al momento de afirmar una de sus premisas. Esto, señalamos, no servía sin embargo para probar que las concepciones eran distintas; Barry podía sostener por medio de otros argumentos la misma conclusión. Aquí es donde entra en juego el segundo contra-argumento ofrecido, que tiene por objeto mostrar que la identidad de concepciones, si es que Barry va a ser consistente con sus afirmaciones con relación a la estabilidad, no puede ser sostenida.

Por el contrario Barry debe reconocer, si el contra-argumento es correcto, no sólo que es incorrecto afirmar la identidad de las concepciones en función de su argumento basado en la identidad del concepto, sino que la concepción de «sociedad bien ordenada», tal y como se la presentaba en «Teoría de la Justicia», implicaba la aceptación por parte de los ciudadanos de una doctrina comprehensiva, que era exactamente lo que se proponía negar.

\section{- 2 -}

2.1- La segunda de las críticas dirigidas por Barry a «Liberalismo Político» sostiene que, al igual que la explicación de la estabilidad que se daba en «Teoría de la Justicia», la ofrecida en esta obra, aunque por motivos distintos, es inadecuada.

Así, mientras que, según Rawls, en «Teoría de la Justicia» la explicación era defectuosa porque implicaba la aceptación por parte de los ciudadanos de una doctrina comprehensiva, en «Liberalismo Político» lo es, según Barry, por redundante o innecesaria.

Antes de pasar al análisis de la crítica de Barry será de utilidad hacer algunas aclaraciones en relación a la manera en que Rawls entiende el problema de la estabilidad.

Una primera precisión consiste en remarcar que Rawls sólo está preocupado por formular una concepción de la justicia dirigida a ordenar sociedades democráticas.

Un segundo punto importante a tener en cuenta es la distinción entre la estabilidad de un régimen democrático y la estabilidad de una concepción política de la justicia.

Señala Rawls en «The Domain of the Political and Overlapping Consensus»:

«Teniendo presente que el tercer hecho general era que un régimen democrático perdurable y estable es aquel que al menos una mayoría sustancial de sus 
ciudadanos políticamente activos libremente sustentan...» (la traducción me pertenece $)^{38}$.

De lo dicho se sigue que la condición suficiente para que un régimen democrático sea estable es que la mayoría de sus ciudadanos lo apoyen libremente. Como se ve, esta es una cuestión empírica. Para determinar si un régimen democrático es o no estable habrá que comprobar si este hecho se da o no.

Una concepción política de la justicia es estable, en cambio, si satisface dos condiciones: que aquellos que crezcan en una sociedad regulada por ella adquieran un «sentido de la justicia», esto es, un deseo de cumplir las exigencias institucionales; que la concepción de justicia que regula las instituciones básicas de esta sociedad bien ordenada se encuentre en un «consenso superpuesto» en relación con las doctrinas comprehensivas razonables que profesan los ciudadanos ${ }^{39}$.

El primer requisito garantiza que los ciudadanos de una sociedad bien ordenada adquieran un sentido de la justicia y tengan por lo tanto un deseo de presentar sus actividades frente a otros como justas.

El segundo requisito garantiza que el sentido de la justicia sea suficiente para motivar la conducta cuando se lo sopesa con otros deseos surgidos a partir de lo que cada individuo considera bueno en función de su particular «doctrina comprehensiva» ${ }^{40}$.

\footnotetext{
38 Rawls, John; «The Domain of the Political and Overlapping Consensus», Op. cit., pág. 479.

39 Señala Rawls en «The Domain of the Political and Overlapping Consensus»: «...una concepción política de la justicia (a diferencia de un régimen político) es estable si satisface las siguientes condiciones: aquellos que han crecido en una sociedad bien ordenada por ella -una sociedad cuyas instituciones son públicamente reconocidas ser justas, de acuerdo a lo especificado por aquella misma concepción- desarrollan una obediencia suficiente a aquellas instituciones, esto es, un sentido de la justicia suficientemente fuerte guiado por los principios e ideales apropiados, de tal manera que ellos normalmente actúan como la justicia lo requiere, a condición de que se les asegure que los demás actuarán de igual manera». Ibid. Dando una exposición más completa del concepto señala en «Liberalismo Político»: «La estabilidad implica dos cuestiones: la primera se refiere a si las personas que se desarrollan entre instituciones justas (tal y como las define la concepción) adquieren normalmente un sentido apropiado de la justicia para que generalmente acaten esas instituciones y cumplan su cometido...». Para agregar luego: «...La segunda cuestión es si, en vista de los hechos generales que caracterizan a una cultura pública democrática, y en particular del hecho del pluralismo razonable, la concepción política puede ser el foco de un consenso traslapado...». Rawls, John; Liberalismo Político, Op. cit., pág. 143.

${ }^{40}$ En el mismo sentido se pronuncia Samuel Freeman señalando las preguntas vinculadas a la estabilidad de una concepción «...Assuming that citizens in a well-ordered society have public knowledge and agreement on justice and just institutions, how do they come to care about them? Rawls contends from the outset that all have a sense of justice and a desire to justify their activities to others as just. In the moral psychology set forth in Chapter 8 of a Theory of
} 
Lo que garantiza, entre otras cosas, que el sentido de la justicia sea $s u$ ficiente es que la concepción política de la justicia sea el foco de un «consenso superpuesto» de doctrinas comprehensivas razonables.

2.2- Pienso que la exigencia de estos dos requisitos está emparentada con la idea de razón práctica bifurcada sustentada por Rawls. Si existen dos poderes de la razón y, consecuentemente, dos ramas de la razón práctica, lo razonable y lo racional; y si estas son complementarias de manera que no pueda entenderse una sin la otra, la explicación de la motivación, y por ende de la estabilidad de la concepción, no está completa hasta que no se ha mostrado cómo es posible, por un lado, que se adquiera el deseo de justificar nuestra conducta ante los demás como correctas, esto es, cómo se adquiere el sentido de la justicia, y por el otro, como este es compatible con lo que consideramos racional a partir de nuestra particular concepción del bien, la cual forma parte de la «doctrina comprehensiva» que profesamos.

El problema que Rawls encontraba en la explicación de la estabilidad presente en «Teoría de la Justicia» se refiere al segundo requisito, esto es, la manera en que allí se explicaba cómo el deseo de actuar conforme al «sentido de la justicia» venía a ser «congruente» con la concepción particular del bien que cada uno de los ciudadanos tenía, y por lo tanto suficiente para motivar la conducta.

En particular Rawls encuentra la explicación que allí se da poco realis$t a$, dado el hecho del pluralismo razonable que caracteriza a cualquier sociedad democrática, puesto que exigía que todos los ciudadanos aceptaran la justicia como imparcialidad basándose en una única doctrina comprehensiva.

En «Liberalismo Político», en cambio, este segundo requisito de la estabilidad no se satisface apelando a una única doctrina comprehensiva, sino a un «consenso superpuesto de doctrinas comprehensivas razonables».

2.3- La crítica de Barry se dirige a mostrar que este recurso, el del «consenso superpuesto», es innecesario puesto que lo que este busca lograr, «...mostrar que personas razonables tienen motivos adecuados para adherir a las demandas de la justicia Rawlsiana...» ${ }^{41}$, ya ha sido alcanzado por el ar-

Justice, he shows how people can come to acquire this disposition to abide by the principles of justice and their requirements in a well-ordered society. This is the first part of the argument for stability in A Theory of Justice(.) But then a second problem arises: even assuming that each person has sense of justice, why should they sufficiently care about justice, to the degree that they recognize and are willing to respect its demands even when these demands conflict with or impede individuals in the pursuit of their conceptions of their good...». Samuel Freeman, «Possibility of a Just Democratic Constitution», en Chicago-Kent Law Review, vol 69, 1994, N³, pág. 625.

41 Barry, Brian; «John Rawls and the Search for Stability», Op. cit., pág. 896. 
gumento que, mediante el recurso de la «posición original», conduce a los principios de justicia.

Si entiendo bien la idea de Barry sería esta: si el objetivo del argumento a favor de la estabilidad es mostrar que los ciudadanos razonables tienen la suficiente motivación de la clase apropiada para cumplir con los principios de justicia, esto se ha logrado con el primer paso del argumento que, a través del recurso de la «posición original» ${ }^{42}$, muestra que los principios allí elegidos son los principios más razonables. Que sean los principios más razonables implica que toda persona que tenga el deseo básico de justificar sus actos ante los demás en principios que ellos, motivados de la misma manera, no podrían rechazar $^{43}$, ya tiene la motivación suficiente.

Ahora bien, continúa el argumento, Rawls acepta como parte de su concepción política el principio de la motivación de Scanlon. Si esto es así, habiendo mostrado con la primera parte del argumento que los principios son razonables, está demostrado también que los ciudadanos razonables que se desarrollen bajo las instituciones organizadas por ellos tendrán la motivación suficiente de la clase adecuada.

Pienso que lo que a Barry le interesa mostrar es, por un lado, que basta tener un «sentido de la justicia» para adquirir un deseo de actuar como aquel requiere porque esto hace innecesario el argumento de la congruencia entre lo justo y lo bueno, y por el otro, que dada la inclusión de la motivación de Scanlon dentro de la concepción rawlsiana de persona razonable, la primera parte del argumento de Rawls basta para sostener que toda persona razonable tiene un «sentido de la justicia».

Esta afirmación se ve sustentada por el hecho de que, según Barry, Rawls se ve conducido al argumento de la congruencia debido a su rechazo de la teoría de la motivación de Ross y Prichard, quienes sostienen que «...reconocer algo como correcto es suficiente para motivar la acción correcta...» (el subrayado me pertenece) ${ }^{44}$.

De este rechazo Barry infiere que la posición de Rawls en este sentido es que existe una brecha entre aceptar los principios de justicia y estar motivados por ellos. Esta es la razón por la que aun si las personas tienen un

42 Es una idea de Barry que si el diseño de la «posición original» está asentado en buenas razones, nada impide pasar directamente de las razones que justifican que el diseño modele las condiciones de razonabilidad a las conclusiones, esto es, a los principios de justicia. Señala al respecto: «Así, puede ser argumentado que los únicos términos que no pueden ser rechazados razonablemente para vivir juntos por los adherentes de cualquier «doctrina comprehensiva» son aquellos corporizados en el primer principio de justicia de Rawls...El segundo principio de justicia se sigue de la premisa de que ningún reclamo válido puede ser hecho en base a ventajas naturales o sociales...». Ibid. pág. 894.

43 Aquí se hace referencia al principio de Scanlon.

44 Barry, Brian; «John Rawls and the Search for Stability», Op. cit., pág. 884. 
«sentido de la justicia», todavía debe mostrarse que este es acorde con el bien de los individuos. ${ }^{45}$

Esto le permite concluir que si el argumento a favor de la congruencia, y en particular el recurso del «consenso superpuesto», tiene por objetivo mostrar que las personas razonables que aceptan los principios de justicia como tales desean conducirse según ellos, esto ya ha sido logrado con la inclusión de la motivación scanloniana y el argumento a partir de la «posición original» que muestra a los principios de justicia como los más razonables.

2.4- No obstante, Rawls nunca ha negado, ni en «Teoría de la Justicia» ni lo hace ahora en «Liberalismo Político», que el tener un «sentido de la justicia» implique tener el deseo de comportarse de acuerdo con los principios de justicia, lo que sí ha negado es que este deseo sea suficiente para motivar la conducta de individuos que poseen dos poderes motivacionales, el que surge de su capacidad de tener un sentido de la justicia, y el que lo hace de su capacidad de tener una determinada concepción del bien ${ }^{46}$.

Planteadas las cosas de esta manera el argumento de Barry parece mostrar que el tener un «sentido de la justicia» ahora, dada la aceptación del principio de Scanlon, implica tener un deseo de comportarse de acuerdo a los principios de justicia, pero esta no es la brecha que el argumento de la estabilidad busca llenar, sino, en cambio, la que existe entre tener un deseo, y que este sea un deseo suficiente para motivar efectivamente la conducta, teniendo en cuenta los deseos que tienen su fuente en el otro poder moral.

Esto explica que la afirmación de Rawls sosteniendo que

«...Aceptar la conexión entre los dos aspectos de lo razonable y el principio de Scanlon equivale a incluir esta forma de motivación en la concepción de las personas razonables a partir de la cual empieza la justicia como imparciali$\operatorname{dad} . . . \gg^{47}$

no lo comprometa con la tesis de que semejante motivación siempre es suficiente, y por lo tanto el recurso del «consenso superpuesto» siga siendo necesario.

${ }^{45}$ Cfr. Ibid. pág. 885.

${ }^{46}$ Lo que Rawls no acepta de la concepción de W.D. Ross es, en primer lugar, «...que el más alto motivo moral es el deseo de hacer lo que es recto y justo, simplemente porque es recto y justo, sin que sea adecuada ninguna otra descripción, y en segundo lugar que, si bien otros motivos tienen, ciertamente, un valor moral, como, por ejemplo, el deseo de hacer lo que es recto porque el hacer esto acrecienta la felicidad humana...estos deseos son menos valiosos, moralmente, que el de hacer lo que es recto sólo porque es recto...». Rawls, John; Teoría de la Justica, Op. cit., pág. 431. Para Rawls, en cambio, es necesario justificar que el deseo de actuar de manera justa tenga prioridad, no basta con postularlo como hace Ross, y esto se logra a través del argumento que muestra el bien del sentido de justicia.

${ }^{47}$ Rawls, John; Liberalismo Político, Op. cit., pág. 67-68 nota 2. 
Creo que lo que se encuentra detrás de la crítica de Barry es por un lado una manera distinta de concebir la razón práctica, y por consiguiente la motivación moral.

Esto lo lleva a pensar que basta que exista un deseo de actuar de manera razonable, para que este ya sea la «motivación suficiente de la clase adecuada»; esto porque, según su punto de vista, la motivación para ser justos es la mera «imparcialidad» entendida como:

«...el deseo de actuar de modo que pueda ser defendido ante uno mismo y ante los demás sin apelar a la ventaja personal...» (el subrayado me pertenece) ${ }^{48}$.

Esta es la razón por la cual Barry sostiene que habiendo Rawls reconocido que las personas razonables tienen el deseo de comportarse de acuerdo al principio de Scanlon, el argumento del «consenso superpuesto» es innecesario.

En efecto, este argumento intenta mostrar que el cumplir con las exigencias de la justicia está sustentado, o por lo menos no es incompatible, con las exigencias que tienen su origen en las «concepciones del bien» que forman parte de sus «doctrinas comprehensivas». Es decir, intenta mostrar que el deseo de cumplir las exigencias de la justicia es acorde, o por lo menos no es contrario, a los deseos que surgen en función de la particular «doctrina comprehensiva razonable» que cada ciudadano profesa.

Para Barry, por supuesto, este paso en el argumento de Rawls es innecesario ya que la motivación para ser justos no debe «apelar a la ventaja personal» y por lo tanto es innecesario mostrar que el deseo de actuar de acuerdo a las exigencias de la justicia es compatible con los deseos que surgen de las «doctrinas comprehensivas razonables» que definen lo que es bueno, y por ende lo que es considerado de valor o ventajoso, para cada persona.

Para Barry la personalidad moral se define por un sólo poder: el de ser imparciales, y consecuentemente la motivación moral está completa si se tiene el deseo de actuar de manera razonable, esto es de acuerdo a términos imparciales que puedan ser defendidos ante uno mismo y los demás sin apelar a la ventaja personal.

Para Rawls, en cambio, la personalidad moral posee dos poderes interrelacionados: la razonabilidad y la racionalidad, o lo que es lo mismo, la capacidad de tener un sentido de la justicia, y la capacidad de tener una determinada concepción del bien. Consecuentemente la motivación moral no está completa si se sostiene que las personas razonables tienen el deseo de actuar de acuerdo a los principios de justicia, es necesario mostrar además que este deseo es congruente, o por lo menos no es incompatible, con los

48 Barry, Brian; Teorías de la Justicia, ed. Gedisa, trad. Cecilia Hidalgo, $1^{\text {a }}$ edición 1995, pág. 378. 
deseos dependientes de las distintas concepciones del bien que las personas razonables pueden profesar en tanto que partes de sus doctrinas comprehensivas.

La motivación moral no está completa hasta que no se ha mostrado cómo pueden quedar comprometidos los dos poderes morales, es decir, hasta que no se ha mostrado que es racional para las personas razonables comportarse de acuerdo a su «sentido de la justicia» ${ }^{49}$.

No basta con sostener que las personas razonables tienen el deseo de comportarse de acuerdo a principios que otras personas motivadas igual que ellas no podrían rechazar. Esta es una parte de la motivación, la que surge de su poder de tener un «sentido de la justicia». Para que este deseo sea suficiente para motivar la conducta es necesario mostrar que existen deseos convergentes, o por lo menos no contrarios, fundados en el otro poder de la personalidad moral, esto es, la capacidad de tener una concepción del bien. Esto se logra a través del argumento del «consenso superpuesto».

Es decir, el argumento de Barry se asienta en una manera distinta de concebir la personalidad moral y, consecuentemente, las exigencias de la razón práctica.

Esto es lo que explica porqué Barry mal interpreta la finalidad del argumento en favor de la congruencia en «Teoría de la Justicia» sosteniendo que este sirve para llenar la brecha que existe entre aceptar los principios de justicia, esto es tener un «sentido de la justicia», y tener el deseo de actuar de acuerdo a ellos. Barry se ve llevado a esto porque para él, si las personas razonables que tienen un «sentido de la justicia» ya tienen también un deseo de actuar según ellos, la motivación moral está completa. Esto lo lleva a pa-

\footnotetext{
49 Pienso que a partir de la publicación de «Liberalismo Político» Rawls sostiene que los ciudadanos en una sociedad bien ordenada poseen dos concepciones de lo bueno, una que forma parte de su doctrina comprehensiva, y otra que forma parte de su concepción política. De esta manera el requisito de la congruencia entre lo justo y lo bueno es ahora visto en un doble sentido. La congruencia entre lo justo y lo bueno como parte de una doctrina comprehensiva es logrado a través del recurso del consenso superpuesto. No obstante si el argumento se detuviera allí la concepción de sociedad bien ordenada sostenida por Rawls quedaría abierta a la objeción que sostiene que esta es vista como un conjunto de individuos que cooperan únicamente para buscar su ventaja, tal como esta es definida a partir de su particular sistema de deseos o preferencias, sin tener fines considerados valiosos en común. En efecto, si lo valioso viene definido sólo por la concepción comprehensiva de cada ciudadano, y si estos no poseen todos la misma, nada puede ser considerado en común como valioso. De esto se encarga el argumento que muestra la congruencia entre lo justo y lo bueno como parte de la concepción política. Todos los ciudadanos en tanto comparten una misma concepción política, comparten también una concepción política del bien, a partir de la cual pueden considerarse ciertas cosas como igualmente valiosas para todos. Dentro de estas valoraciones compartidas o fines comunes se encuentra el bien de la sociedad política en sí misma, y no como medio para alcanzar objetivos personales. Cfr. Rawls, John; «Justice as Fairness: A Restatement», Op. cit., pág. 198-202.
} 
sar por alto que tanto la finalidad del argumento de la congruencia en «Teoría de la Justicia», como el del «consenso superpuesto» en «Liberalismo Político» es mostrar que, teniendo en cuenta los dos poderes motivacionales de la personalidad moral, el deseo de actuar conforme a los principios de justicia es normalmente suficiente para motivar efectivamente la conducta.

Para que el argumento funcione en contra de Rawls, no sólo en contra de su argumento a favor de la estabilidad, sino también en contra de toda la construcción de la «posición original», sería necesario mostrar que su concepción de la razón práctica y la personalidad moral es la más adecuada. Sin embargo no hay nada en el argumento de Barry que tienda a mostrar esto. Su crítica a Rawls no se presenta como una crítica externa a su teoría, sino como aceptando sus propias concepciones, y como tal, por lo señalado, es insostenible.

\section{Conclusión}

Las críticas de Barry a Rawls podrían sintetizarse en dos palabras: inconsistencia y redundancia. Inconsistencia en tanto que, al sostener que «Teoría de la Justicia» era o formaba parte de una «doctrina comprehensiva» Rawls no mantenía la noción que de este tipo de doctrinas formulaba en «Liberalismo Político». Redundancia en tanto que la solución que se ofrecía en «Liberalismo Político» al problema de la defectuosa explicación presente en «Teoría de la Justicia» de la estabilidad de la concepción en base a una «doctrina comprehensiva» compartida era innecesaria.

Estas críticas, como he señalado, son internas a la justicia como imparcialidad, es decir no cuestionan sus presupuestos y categorías sino que se asientan en ellos. Así, por ejemplo, no es el objetivo de Barry cuestionar la noción de «doctrina comprehensiva» utilizada por Rawls, sino mostrar que el uso que se hace de esta noción al sostener que «Teoría de la Justicia» era o formaba parte de una doctrina tal es inadecuado. Tampoco Barry cuestiona la noción de estabilidad de una concepción política expuesta por Rawls, sino que señala que dando por sentada esta noción, el argumento que tiende a mostrar la estabilidad de la justicia como imparcialidad no necesita hacer uso del recurso del «consenso superpuesto».

Como el trabajo es un análisis de las críticas de Barry, y estas tienen el carácter antes señalado, no hay nada en él que sirva como argumento acerca de la corrección de la posición de Rawls. Un argumento en tal sentido tendría que referirse a los presupuestos mismos de la concepción de la justicia y no sólo a la correcta interconexión de sus elementos.

Sin embargo, pienso que la tarea no por menor es innecesaria. Esto porque sólo tiene sentido preguntarse por la corrección de una teoría si esta no es defectuosa en su estructura. 
Puede afirmarse entonces, por las razones expuestas, que «Teoría de la Justicia» contenía genuinos defectos internos, el carácter comprehensivo de la concepción y la explicación de la estabilidad en base a una doctrina de este tipo, y que «Liberalismo Político» al intentar subsanarlos, a través del recurso del «consenso superpuesto», no ha incurrido en defectos semejantes. 